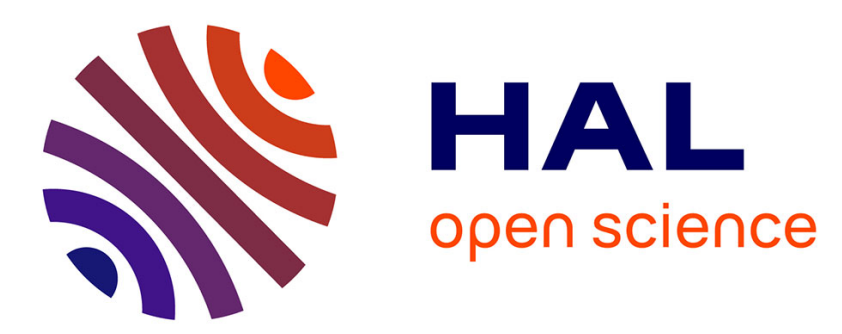

\title{
A Novel Deep Learning Approach for Liver MRI Classification and HCC Detection
}

Rim Messaoudi, Faouzi Jaziri, Antoine Vacavant, Achraf Mtibaa, Faïez Gargouri

\section{- To cite this version:}

Rim Messaoudi, Faouzi Jaziri, Antoine Vacavant, Achraf Mtibaa, Faïez Gargouri. A Novel Deep Learning Approach for Liver MRI Classification and HCC Detection. A Novel Deep Learning Approach for Liver MRI Classification and HCC Detection, pp.635-645, 2020, 10.1007/978-3-030-59830-3_55 . hal-03135435

\section{HAL Id: hal-03135435 \\ https://hal.uca.fr/hal-03135435}

Submitted on 9 Feb 2021

HAL is a multi-disciplinary open access archive for the deposit and dissemination of scientific research documents, whether they are published or not. The documents may come from teaching and research institutions in France or abroad, or from public or private research centers.
L'archive ouverte pluridisciplinaire HAL, est destinée au dépôt et à la diffusion de documents scientifiques de niveau recherche, publiés ou non, émanant des établissements d'enseignement et de recherche français ou étrangers, des laboratoires publics ou privés. 


\title{
A Novel Deep Learning Approach for Liver MRI Classification and HCC Detection
}

\author{
Rim Messaoudi ${ }^{1,2[0000-0001-5311-0375]}$, Faouzi Jaziri ${ }^{2[0000-0002-1417-2458]}$, Antoine Vacavant ${ }^{2[0000-}$ \\ 0001-9616-3282], Achraf Mtibaa ${ }^{1,3[0000-0002-4397-1793]}$ and Faïez Gargouri1,4[0000-0003-2575-8654] \\ ${ }^{1}$ MIRACL Laboratory, University of Sfax, Sfax, Tunisia \\ ${ }^{2}$ Institut Pascal, Université Clermont Auvergne, UMR6602 CNRS/ \\ UCA/SIGMA, 63171 Aubière, France \\ ${ }^{3}$ National School of Electronic and Telecommunications, University \\ of Sfax, Sfax, Tunisia \\ ${ }^{4}$ Higher Institute of Computer Science and Multimedia, University of
} Sfax, Sfax, Tunisia

\begin{abstract}
This work proposes a deep learning algorithm based on the Convolutional Neural Network (CNN) architecture to detect HepatoCellular Carcinoma (HCC) from liver DCE-MRI (Dynamic Contrast-Enhanced MRI) sequences. The Deep Learning technique is an artificial intelligence technique (AI) that tries to imitate the human brain work in the training data and creating models used for decision. Actually, it is widely used for various clinical issues. To diagnose HCC, radiologists consider three different phases during contrast injection (before injection; arterial phase; portal phase for instance). This paper presents an approach that offers a parallel preprocessing algorithm. It allows HCC detection and localization in MRI images via a CNN algorithm. The created CNN model reached an accuracy level of $90 \%$ in both arterial and portal phases using MRI patches of $64 \times 64$ pixels. We mention also its ability to decrease false detection comparing with our previous works. The obtained good accuracy is considered to be ameliorated in our future works.
\end{abstract}

Keywords: Medical image analysis, HCC, Deep learning, CNN classification, MRI

\section{Introduction}

Liver cancer begins by affecting healthy cells and forming a mass presenting a tumor. Liver cancer is considered the sixth most common cancer worldwide. According to the Adrienne Wilson Liver Cancer Association ${ }^{1}$, this year there will be over 42,030

${ }^{1}$ https://www.bluefaery.org/statistics/ 
(29,480 men and 12,550 women) new cases of liver cancer and around 31,780 (21,600 men and 10,180 women) deaths. HCC (HepatoCellular Carcinoma) is considered the most dangerous liver cancer according to the World Health Organization $(\mathrm{WHO})^{2}$.Diagnosis of this cancer is used through different modalities such as MRI, CT scans and Ultrasound. The follow-up of HCC is defining the high-risk patient. Male gender, older age, alcohol, smoking, family history of HCC, and the cirrhosis are also among risks factors regardless of different regions[1][2]. The American Association for the Study of Liver Diseases (AASLD), the Asian-Pacific Association for the Study of the Liver (APASL), and the European Association for the Study of the Liver (EASL) societies declared the importance of liver cancer surveillance with abdominal imaging such as MRI and CT scans each 6 months [3].

HCC detection is a very challenging task, and has been treated in several research works. In this context [4] proposed an automatic CNN with Multi-Magnification Input Images. The algorithm is based on two main steps: a) the extraction of cell level and structure features maps from high and low-magnifications images respectively by separating general convolutional networks, and b) the integration of multimagnification features by the use of fully connected network. [5] proposed a method that classifies common hepatic lesions on multi-phasic MRI. This classification approach is realized through different steps: a) crop and resize a set of images around each tumor, b) split randomly lesions into train and test sets, c) create 100 copies of each image presenting a lesion through various image processing techniques, d) using the training set to associate image patterns with the adequate lesion class, e) validation of the model by using the test set and f) evaluating the model performance. In addition,[6]integrated deep learning into MRI image and proposed a framework for HCC lesions detection from DCE-MRI (Dynamic Contrast-Enhanced MRI) sequences. This approach has employed the U-Net architecture in the classification process. [7] proposed a parallel Framework for HCC detection in DCE-MRI sequences with wavelet-based description and SVM (Support Vector Machine)classification. This work realized parallel patch-based processing of DCE-MRI images characterizing three contrast-enhancement phases. In addition, authors used a wavelet-based descriptor, presenting the local signal evolution of slices during DCE-MRI acquisition process, extracted in a SVM classifier.[14]presented an approach that has generated synthetic medical images applying deep learning GANs (Generative Adversarial Networks). This approach is used to improve the performance and the effectiveness of $\mathrm{CNN}$ for medical image classification.

All the previous cited works have improved the importance of applying classification methods to classify medical images and data. Although, they do not deeply used CNN to classify liver MRI images especially for CNN and show its effectiveness to reach high measures of accuracy. In this paper, we propose a HCC detection framework that uses CNN architecture. In fact, we have developed a novel algorithm that proposes 1) a parallel preprocessing algorithm. It takes as input MRI images and

${ }^{2}$ https://www.who.int/fr 
gives fragments with equal sizes as output, and 2) creates a parallel CNN algorithm that allows HCC detection and localization in MRI images. The goal here is to increase the Dice (or F-measure, or F1) compared to our previous works $[6,8]$ by trying to keep an interesting accuracy rate. Dice coefficient is considered as an important measure in medical imaging. It is used to gauge the similarity degree of two samples and this is efficient in our case because input classes have not the same size. The rest of the paper is organized as follows: Section 2 presents the material employed in this work. Section 3 explains the proposed approach. Section 4 exposes the obtained results and discusses and Section 5 concludes the paper and gives an overview of the future works.

\section{$2 \quad$ Material and Method}

\subsection{Data-set}

In this work, we have used 9 patients who underwent hepatic MRI in our department as standard of care for their cirrhosis. They all suffer from HCC diagnosed in the MRI by a radiologist, according to EASL criteria [9]: known cirrhosis (according to clinical, biological and imaging data), focal lesion of at least $1 \mathrm{~cm}$, hypervascular in the arterial phase with washout in the portal venous or delayed phases. MR exams were performed on a $1.5 \mathrm{~T}$ Optima (General Electric Healthcare, Milwaukee, WI) with a phased array coil. It consisted of axial T2 Single Shot Fast Spin Echo (SSFSE), axial T2 Fat Sat Propeller, axial diffusion weighted imaging and multiphase 3D Fast Spoiled Gradient Echo T1 LAVA before and while injection of $0.2 \mathrm{~mL} / \mathrm{kg}$ of gadobenate dimeglumine (MultiHance ${ }^{\circledR}$, Bracco).MR images were obtained in the axial plane with a section thickness of $4 \mathrm{~mm}$, a $2 \mathrm{~mm}$ intersection gap, matrix of $512 \times 512$ and a field of view of $420 \times 420-500 \times 500 \mathrm{~mm} 3$, which covered the whole liver. In this study, we have applied 2D (slice by slice) data, only $\mathrm{T} 1$ images were used to detect HCC.

\subsection{Liver Segmentation}

Liver segmentation from MRI images is important in the process of tumor detection. In this work, we have applied the segmentation model developed by [7, 13]. The method consists of developing an automatic model for both modalities MRI and CT scans. In the first step, authors extracted 4 different statistical models with 68 livers segmented by clinical experts obtained from Shape 2015 [10], IRCAD [11] and SLIVER07 [12] databases. The obtained 4 models were constructed according to their variabilities from a standard shape liver. Therefore, statistical model and all patient volumes have equivalent dimensions. Then, we localize the liver on the images with the mean dimensions of a standard liver. This localization of the liver allows us to compute a threshold to isolate pixels that belong to the liver. After the thresholding on 
each slice, we apply a contour enhancement process. At this step we use the model the liver as a probability map to localize the liver and we then perform an active contoursegmentation method (fast marching) resulting in a binary mask. Finally, to erase errors due to over-segmentation by a process considering the global shape of the liver. The steps of the segmentation method are presented in Figure 1.

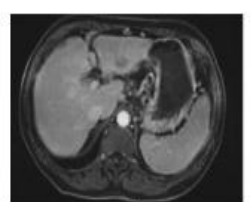

(a)

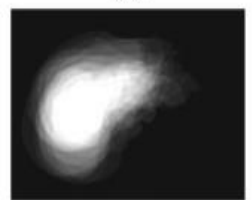

(d)

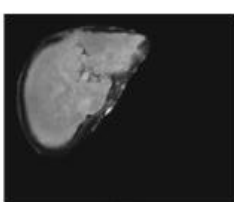

(b)

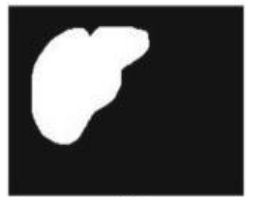

(e)

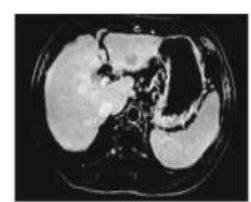

(c)

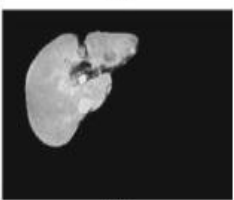

(f)

Fig.1. Liver segmentation steps: a) a patient slice, b) the largest liver surface (threshold computation), c) thresholding and contour enhancement, d) liver model for localization and active contour method, e) the obtained mask, and f) the final segmentation result. 


\section{Deep Learning for HCC Detection by CNN}

In this section, we present our deep learning approach. We will give an overview of the different steps as well as the generated contributions. Our approach is composed of five main steps; 1) Pre processing 2) Training phase 3) Prediction Phase, 4) Testing phase and 5) the validation phase. Figure 2 shows our Deep Learning workflow.

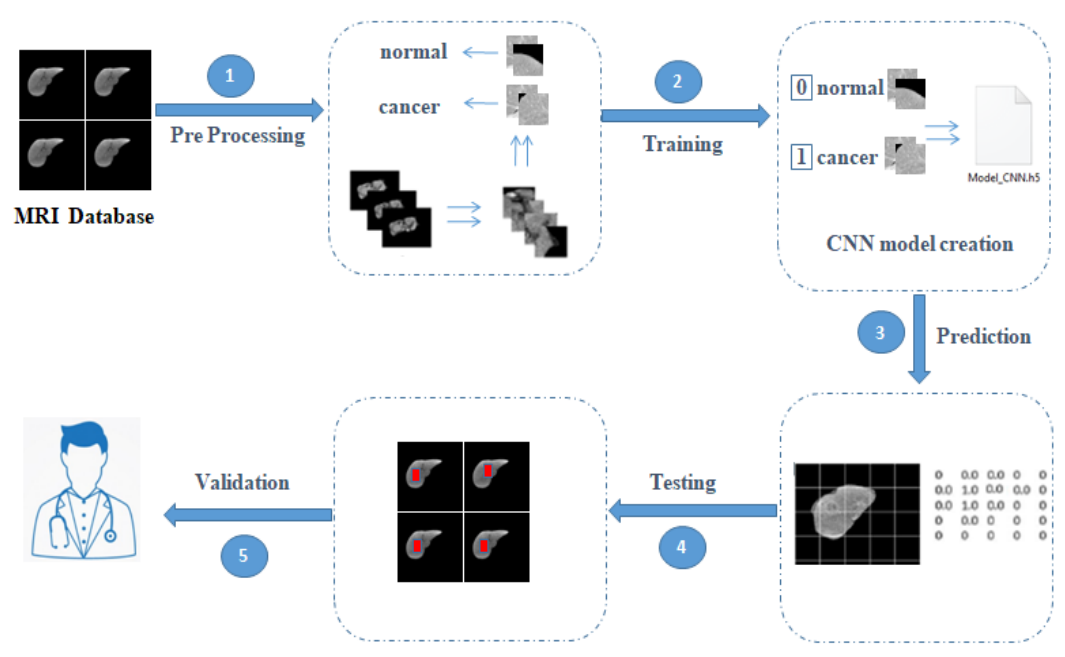

Fig.2. Liver tumor detection and localization approach

\subsection{Pre-Processing Step}

This step focuses on images preparation and manipulation. These images are useful later to prepare the databases. During the MRI process, the radiologist or the doctor takes three different MRI images for each patient, depending on the time after the contrast injection. This will take into account the evolution of information in these different image sequences. Figure 3 exposes an example of these images. (a) Phase 1: without injection, (b) Phase 2: short time of (30s-1min) after the contrast injection, and (c) Phase 3: long time (2-5min) after the contrast injection. 


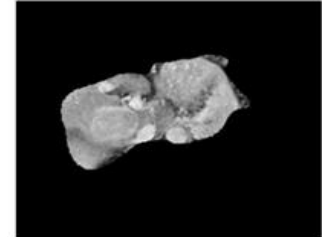

(a)

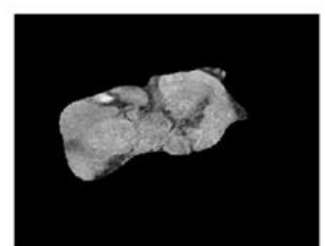

(b)

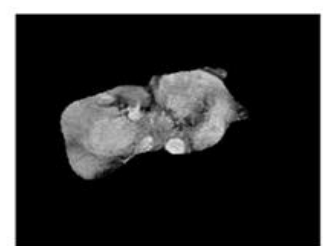

(c)

Fig.3. MRI images in the three phases

Based on a well-defined fragment size; $64 \times 64$ pixels in our case defined as follows; each block size is $58 \times 58$ with a 3-pixel overlap in the 4 directions. We perform a division of MRI images of several patients. Figure 4 shows an example of one image division. In the case of the image size is smaller than the size of the fragment, we use resizing mechanisms. In our case, we have applied the padding technique. We add values from 0 to the end of the matrix until we wait for a new size matrix for the fragment. Moreover, our program eliminates each fragment that admits a total medium of intensity equal to 0 : it is the case we have a fragment with only a black background.We have used later the different fragments resulting from this to create Deep Learning databases.

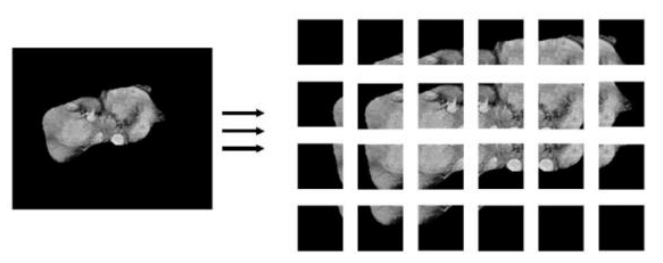

Fig.4. MRI images fragments 


\subsection{Training phase}

The ability to learn in the Deep Learning algorithm has a very important feature, but this technique requires a large computing time and several training information. This algorithm follows a well-defined learning technique, it learns from the tagged information. Hence, we have input variables (x) and output variables (Y) and we use an algorithm to execute a mapping function between input and output. The goal is to approximate the mapping function so that when we have new input data (x), we can predict the output variables (Y) for that data. The proposed model is a consecutive of convolution blocks dedicated to extract and identify images features. These images are interconnected with a fully connected block that classifies fragments. In order to create the model and implement the fragment database, we have initialized the different relevant variables in our algorithm; the epoch number was fixed in the value 100. We have initialized 41,794 steps per epoch for the train and 6,000 steps for validation. We have used another variable "CallBacks" which interrupts the training if there is no change in the error value. This value shows the difference between the results obtained after the forward propagation phase and the results introduced as an input to the algorithm. In addition, we have fixed the Batch size on the value 10. This variable helps to minimize the time of calculation since it shows the number of images to use during a forward propagation cycle. Weight and bias values are initialized in a randomly. Figure 5 shows the proposed Deep Learning model.

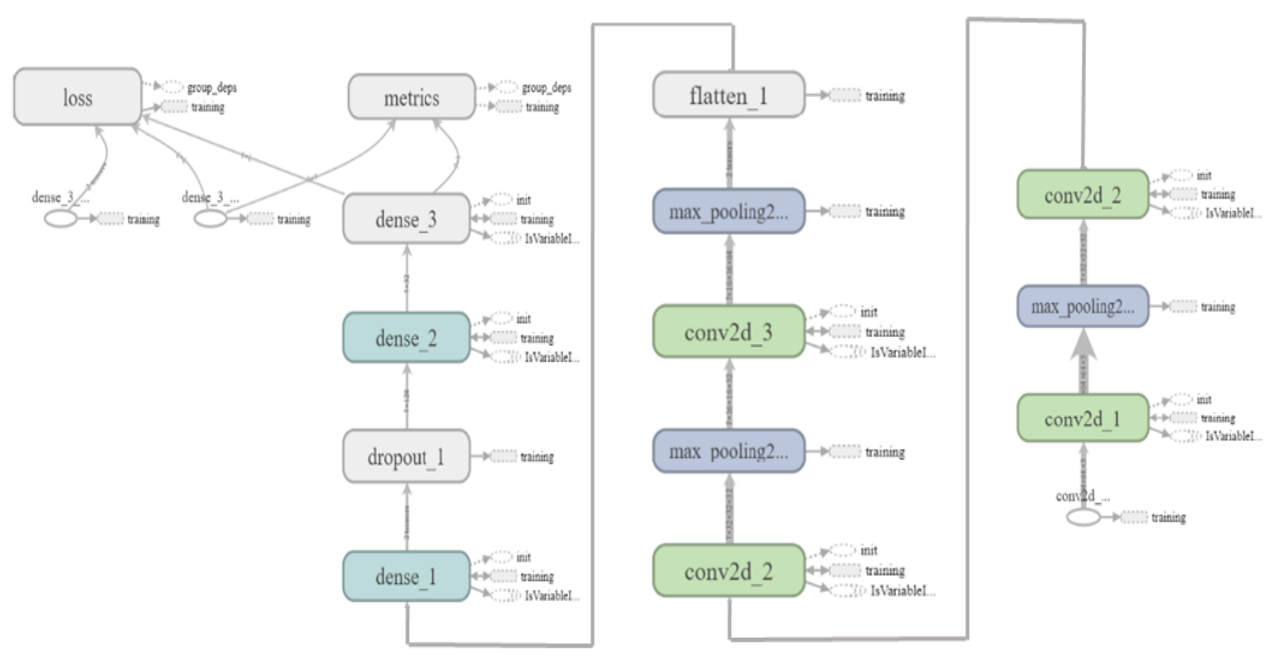

Fig.5. The proposed Deep Learning model 
We perform various convolutions on our model, where each task uses an alternative filter. After the convolution phase, we use an activation function to achieve a nonlinear output. It is the ReLU activation function. Finally, features go through a last block called Pooling which reduces the size of the map. The last step is the model registration. Indeed, the model is saved under the extension (.h5) because it characterizes structured format that supports a large mass of information.

\subsection{Prediction Phase}

After the training phase and the recording of the model, we have prepared new images for the prediction. Such an image is divided into non-overlapping 64x64 fragments and we predicted each fragment independently of the others. As shown in Figure 25, the value 0 is attributed to a normal fragment and the value 1 indicates that the fragment potentially contains a tumor, since each fragment name has its abscissa and its ordered in the source image, we can build this matrix where each value represents the prediction of the corresponding fragment. Figure 6 presents an example illustrating the prediction phase description.
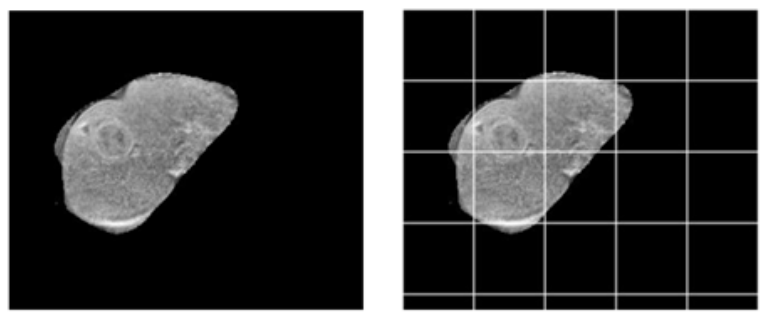

$\begin{array}{lllll}0 & 0.0 & 0.0 & 0 & 0\end{array}$

$\begin{array}{lllll}0.0 & 1.0 & 0.0 & 0.0 & 0\end{array}$

$\begin{array}{lllll}0.0 & 1.0 & 0.0 & 0 & 0\end{array}$

$\begin{array}{lllll}0 & 0.0 & 0 & 0 & 0\end{array}$

$\begin{array}{lllll}0 & 0 & 0 & 0 & 0\end{array}$

Fig.6.The prediction phase description

\subsection{Testing and validation Phases}

In these steps, we test the efficiency of our algorithm by using different images related to different real patient's cases. More details about the carried out results are mentioned in the next section. 


\section{$4 \quad$ Results}

\subsection{Applied Tools}

To develop our approach, we have used Python ${ }^{3}$ which offers a dynamic system and automatic memory management. It supports several programming paradigms, including object-oriented, imperative, functional programming and procedural styles. It promotes structured, functional and object-oriented imperative programming. We have used also various libraries and packages such as Tensor Flow ${ }^{4}$ which is an open source platform for machine learning. It is a comprehensive, flexible ecosystem of libraries and community resources. Also, Keras ${ }^{5}$ is applied in our Deep Learning model. It is a high-level neural networks API and enabling fast experimentation. Numpy ${ }^{6}$ is the fundamental API for computing with Python language. It contains a powerful $\mathrm{N}$-dimensional array object and various tools for integrating $\mathrm{C} / \mathrm{C}++$ and Fortran code. Open $\mathrm{CV}^{7}$ is also applied in our approach. It is an open graphic library, developed by Intel and applied in real time images processing. To run the deep learning algorithm, we have used Google Colaboratory Notebook because it provides a totally free GPU and other free cloud services.

\subsection{Database Description}

The used database, as mentioned in Table 1, is composed of a set of fragments, divided as follows; for the training phase, we have used 26,162 normal patches and 15,632 cancerous patches. For the Testing phase, we have used 3,588 normal patches and 2,412 cancerous patches. The size of each patch is $64 \times 64$ pixels.

Table1.Deep Learning database Description

\begin{tabular}{|l|l|l|l|}
\hline Database (number of patches) & Normal & cancer & Total \\
\hline Training Data & 26,162 & 15,632 & 41,794 \\
\hline Testing Data & 3,588 & 2,412 & 6,000 \\
\hline Total & 29,750 & 18,044 & 47,794 \\
\hline
\end{tabular}

\footnotetext{
3 https://www.python.org/

4 https://www.tensorflow.org/

5 https://keras.io/

6 https://numpy.org/

7 https://opencv.org/
} 


\subsection{Performance metrics}

The most commonly used evaluation methods for a Deep Learning model are mentioned in Table 2. Hence, we have used a set of measures of performance such as Sensitivity (SEN), Accuracy (ACC), Specificity (SPC), and F1 score.

Table2.Performance metrics

\begin{tabular}{|l|l|}
\hline TP & True Positive \\
\hline TN & True Negative \\
\hline FP & False Positive \\
\hline FN & False Negative \\
\hline SEN & TP/TP+FN \\
\hline ACC & TP/TP+FP \\
\hline SPC & TN/(TN+FP) \\
\hline F1 Score & $(2 *$ Recall $*$ Precision $) /($ Recall + Precision $)$ \\
\hline
\end{tabular}

\subsection{Experimental Results}

The proposed HCC liver cancer classification model was implemented with Python with system configurations such as an i7 processor with 8 GB RAM. The training of the Deep Learning algorithm requires a very important computing time, we have integrated the TenserFlow Framework to accelerate computing time. The execution of the algorithm with Google Collaboratory Notebook reached 98\% (Fig.5 a)and the error rate was 0.03 (Fig. 5 b).Tables 3 and 4 demonstrate respectively the performance levels of liver cancer MRI classification rates for the proposed approach. In this test, we have taken the 9 patients of our dataset and we tried to predict the existence of HCC tumors using our model. The proposed model provides better classification results. 


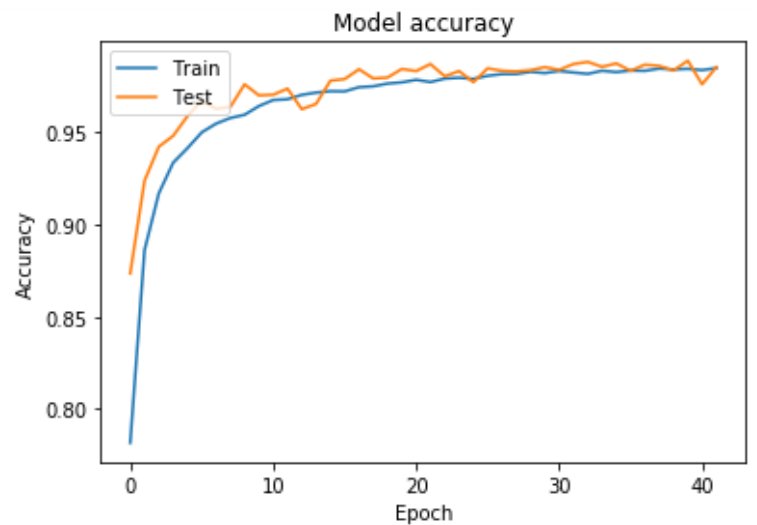

(a)

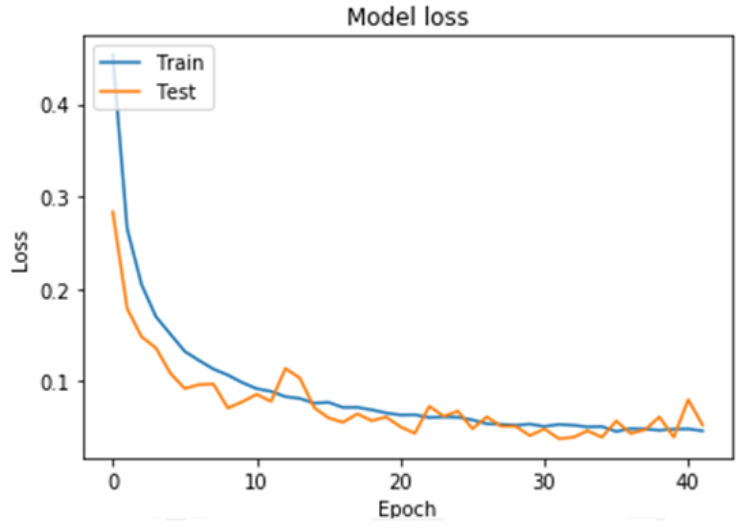

(b)

Fig.7.The proposed Deep Learning model: (a) accuracy rate, (b) error rate

Results obtained by our model were illustrated respectively in tables 3 and 4 for both phases 2 (With Contrast) and 3 (After contrast).To evaluate our model, we have used the same datasets employed in works [6,8]. We have applied the SVM method in [6] and U-Net in [8] while we have applied CNN in this work. The performance of the proposed CNN model is determined by its ability to detect cancerous and normal patches from MRI images. We have used the same evaluation criteria as our previous works. Hence, based on the obtained findings, the model is able to predict the medical status of the liver. The accuracy has clearly indicated that the proposed algorithm is deeply efficient in detecting HCC slices. For the phase 2, results were $80 \%$ (SEN), $71 \%$ (Acc), 92\% (SPC) and 74\% (F1 score). For phase 3, results were; $81 \%$ (SEN), $68 \%$ (Acc), 90\% (SPC) and 73\% (F1 score). The total accuracy level reached $90 \%$ in both phases. Regarding our previous studies [6] and [8], F1 score level has been increased and reached respectively $74 \%$ and $73 \%$ for phase 2 and phase 3 considering [6].This rate is the most suitable and adapted measure to evaluate our model because 
the used classes have not the same size. In fact, F1 score has been increased from $66.5 \%$ in [8] to $74 \%$ in our work. In addition, based on results obtained in [6], Sensitivity rate has been increased in phase 3 from $76 \%$ to $81 \%$. Also, Specificity rates have been increased respectively in both phases 2 and 3 from $87 \%$ and $82 \%$ to $92 \%$ and $90 \%$ in our case.

Table3.With-Contrast Phase Evaluation

\begin{tabular}{|c|c|c|c|c|c|c|c|c|c|c|}
\hline & Patient & Patient & Patient & Patient & Patient & Patient & Patient & Patient & Patient & Total \\
& 1 & 2 & 3 & 4 & 5 & 6 & 7 & 8 & 9 & \\
\hline TP & 6 & 3 & 6 & 30 & 116 & 5 & 98 & 27 & 163 & 454 \\
\hline TN & 65 & 17 & 38 & 169 & 454 & 40 & 503 & 43 & 628 & 1957 \\
\hline FP & 14 & 0 & 5 & 11 & 22 & 5 & 70 & 2 & 31 & 160 \\
\hline FN & 4 & 0 & 6 & 0 & 10 & 0 & 65 & 5 & 15 & 105 \\
\hline SEN & 0.5 & 1 & 0.5 & 1 & 0.92 & 1 & 0.6 & 0.84 & 0.91 & 0.80 \\
\hline ACC & 0.5 & 1 & 0.5 & 0.73 & 0.84 & 0.5 & 0.58 & 0.93 & 0.84 & 0.71 \\
\hline SPC & 0.87 & 1 & 0.88 & 0.93 & 0.95 & 0.88 & 0.87 & 0.95 & 0.95 & 0.92 \\
\hline F1 Score & 0.5 & 1 & 0.5 & 0.84 & 0.87 & 0.66 & 0.59 & 0.88 & 0.87 & 0.74 \\
\hline
\end{tabular}

Table4.After-Contrast Phase Evaluation

\begin{tabular}{|l|c|c|c|c|c|c|c|c|c|c|}
\hline & Patient & Patient & Patient & Patient & Patient & Patient & Patient & Patient \\
7 & 2 & 3 & 4 & 5 & $\begin{array}{c}\text { Patient } \\
9\end{array}$ & $\begin{array}{c}\text { Total } \\
7\end{array}$ \\
\hline TP & 6 & 3 & 6 & 17 & 111 & 5 & 109 & 24 & 159 & 440 \\
\hline TN & 67 & 17 & 38 & 62 & 454 & 40 & 537 & 44 & 618 & 1877 \\
\hline FP & 12 & 0 & 5 & 9 & 21 & 5 & 51 & 6 & 32 & 141 \\
\hline FN & 4 & 0 & 6 & 1 & 14 & 0 & 48 & 3 & 27 & 103 \\
\hline SEN & 0.6 & 1 & 0.5 & 0.94 & 0.88 & 1 & 0.69 & 0.88 & 0.85 & 0.81 \\
\hline ACC & 0.33 & 1 & 0.54 & 0.65 & 0.84 & 0.5 & 0.68 & 0.8 & 0.83 & 0.68 \\
\hline SPC & 0.84 & 1 & 0.88 & 0.87 & 0.95 & 0.88 & 0.91 & 0.88 & 0.95 & 0.90 \\
\hline F1 Score & 0.42 & 1 & 0.52 & 0.77 & 0.86 & 0.66 & 0.68 & 0.84 & 0.84 & 0.73 \\
\hline
\end{tabular}

\section{Conclusion}

This article discusses the topic of HCC detection through deep learning CNN architectures. It proposed a Deep Learning Approach for Liver MRI Classification and HCC Detection The proposed approach has offered a preprocessing script that provides the necessary information for the entry of the Deep Learning algorithm. Also, it creates a parallel Deep Learning Algorithm that targets the detection and localization of tumors in a medical liver image. Results have shown a better classification in case of liver MRI Images compared with others classification techniques, in term of F1 measure, but still with a high accuracy. Moreover, applying an automatic CNN classification approach achieved better precision and accuracy levels especially in the third phase which reached $90 \%$. Moreover, according to the experimental findings, the 
proposed approach is effective for the classification of the human liver MRI images in terms of accuracy, sensitivity, and specificity. As future works, we aim at ameliorating the performance level of our algorithm by including other semantic techniques in the detection process.

Acknowledgement. This work was financially supported by the "PHC Utique" program of the French Ministry of Foreign Affairs and Ministry of higher education and research and the Tunisian Ministry of higher education and scientific research in the CMCU project number 18G1139 - Campus France Code 39319SM.

\section{References}

1. Bialecki, ES., and Di Bisceglie, AM.: Diagnosis of hepatocellular carcinoma. HPB (Oxford) 7(1), 26-34 (2005)

2. Ghouri, YA., Mian I., Rowe JH.:Review of hepatocellular carcinoma: Epidemiology, etiology, and carcinogenesis. Journal of Carcinogenesis 16 (1), (2017)

3. Ayoub, W.S., Steggerda, J.,Yang, J.D., Kuo, A., Sundaram, V., and Lu.: Current status of hepatocellular carcinoma detection: screening strategies and novel biomarkers. Therapeutic Advances in Medical Oncology 11 (2019)

4. Huang WC., Chung PC., Tsai HW., Chow NH., Juang YZ., Tsai HH., Lin SH., Wang, CH.: Automatic HCC Detection Using Convolutional Network with Multi-Magnification Input Images. In. IEEE International Conference on Artificial Intelligence Circuits and Systems (AICAS), pp 194-198, IEEE, Hsinchu, Taiwan (2019)

5. Charlie, A. Hamm, Clinton J. Wang, Lynn J. Savic, Ferrante, M, Schobert, I, Schlachter T, De Lin M., Duncan, JS., Weinreb, JC., Chapiro, J., and Letzen. B.: Deep learning for liver tumor diagnosis part I: development of a convolutional neural network classifier for multiphasic MRI. European Radiology 29 (7), 3338-3347 (2019)

6. Fabijańska, A., Vacavant, A., Lebre MA., L. M. Pavan A., de Pina DR., Abergel A., Chabrot, P., Magnin, B.U-CatcHCC: An accurate HCC detector in hepatic DCE-MRI sequences based on an U-Net framework. In. International Conference on Computer Vision and Graphics, ICCVG 2018, Warsaw, Poland, 11 pages (2018)

7. Lebre, MA., Arrouk, K., V〜o V־an, A.-K, Leborgne, A., Grand-Brochier, M., Beaurepaire, P., Magnin, AB., Abergel, A., and Chabrot, P.:Medical Image Processing and Numerical Simulation for Digital Hepatic Parenchymal Blood Flow. In Simulation and Synthesis in Medical Imaging, workshop of MICCAI 2017 (LNCS 10557).Springer, 99-108 (2017)

8. Pavan, A. L. M., Benabdallah, M., Lebre,M.A., Rodigrues de Pina, D., Jaziri, F., Vacavant, A., Mtibaa, A., Mohamed Ali, H., Grand-Brochier, M. Rositi, H, Magnin, B., Abergel, A., and Chabrot, P.:A Parallel Framework for HCC Detection in DCE-MRI Sequences with Wavelet-Based Description and SVM classification. In. Proceedings of the 33rd Annual ACM Symposium on Applied Computing, pp. 14-21, Pau, France,(2018)

9. European Association for the Study of the Liver. 2012. EASL-EORTC Clinical Practice Guidelines: Management of hepatocellular carcinoma. Journal of Hepatology, 56(4)908943 (2018)

10. Kistler, M., Bonaretti, S., Pfahrer M., Niklaus, R., and Büchler P.:The virtual skeleton database: an open access repository for biomedical research and collaboration. Journal of Medical Internet Research15(11), e245 (2013) 
11. Research Institute against Digestive Cancer. IRCAD dataset. http://www.ircad. $\mathrm{fr} / \mathrm{research} / 3 \mathrm{~d}$-ircadb-01/

12. Heimann, T., et al.: Comparison and evaluation of methods for liver segmentation from CT datasets. IEEE Trans. Med. Imaging 28(8), pp.1251-1265,(2009)

13. Lebre,M.-A., Vacavant, A., Grand-Brochier, M., Strand, R., Rosier, H., Abergel, H., Chabrot, P., Magnin, B.: A robust multi-variability model based liver segmentation algorithm for CT-scan and MRI modalities. Computerized Medical Imaging and Graphics, 76,11 pages, ( 2019)

14. Frid-Adar, M., Diamant, I., Klang, E., Amitai, M., Goldberger, J., and Greenspan H.: GAN-based synthetic medical image augmentation for increased CNN performance in liver lesion classification. Neurocomputing 321, 321-331, (2018) 